\title{
A Clinicopathological Correlation of Pineal Region Tumours
}

\section{Prabal Deb ${ }^{1}$ and Gurpreet Kaur ${ }^{2 *}$}

${ }^{1}$ Director Operations and Chief Histopathologist SRL Diagnostics, India

${ }^{2}$ Department of Hematopathology, All India Institute of Medical Sciences, India

*Corresponding Author: Gurpreet Kaur, Senior Resident, Department of Hematopathology, All India Institute of Medical Sciences, New Delhi, India.
Received: September 16, 2020

Published: October 15, 2020

(C) All rights are reserved by Prabal Deb and Gurpreet Kaur.

\begin{abstract}
A variety of lesions involve the pineal region these include both neoplastic processes and congenital lesions. Tumors of the pineal region are classified into those arising from the pineal parenchyma, germ cell neoplasms, metastasis, and lesions arising from adjacent structures. They make up less than $1 \%$ of intracranial tumors in adults and are commoner in children. Knowledge of the variety of lesions that occur in the pineal region, their imaging appearances, and their clinical features assists in narrowing the differential diagnosis and optimizing patient treatment of these rare tumors. Pineal gland lesions as it is are rare, with only a few cytologic descriptions occurring in the literature. We hereby present 04 cases of pineal region tumours with a radiology cytology and histopathological correlation.
\end{abstract}

Keywords: Pineal Gland; Cytology; Pineoblastoma; Pineocytoma; Germinoma

\section{Abbreviations}

WHO: World Health Organization; PPTID: Pineal Parenchymal Tumor of Intermediate Differentiation; PTPR: Papillary Tumor of the Pineal Region; LSAB: Labeled Streptavidin Biotin; HPE: Histopathological Examination; NSE: Neuron Specific Enolas; AFP: Serum Alpha Fetoprotein; CEA: Carcinoembryonic Antigen; hCG: Human Chorionic Gonadotropin; CK: Cytokeratin; GEAP: Glial Fibrillary Acidic Protein; HPF: High Power Field

\section{Introduction}

The pineal gland is a small reddish-brown structure that derives its name from its pinecone-like shape. The pineal ranges in size from 10 to $14 \mathrm{~mm}$; it is located in the midline, above the tentorium and superior colliculi and below the splenium of the corpus callosum and the vein of Galen, and is attached to the superior aspect of the posterior border of the third ventricle. Histologically, lobules of pineocytes (95\%) and astrocytes (5\%) separated by a fibrovascular stroma make up the normal gland. The pineal gland does not have a blood-brain barrier and therefore enhances on contrast material-enhanced images.

Pineal gland tumors are rare and account for less than $1 \%$ of all primary brain tumor diagnosis. Also, they are more commonly seen in children, accounting for 3\% - 8\% of intracranial neoplasms in the pediatric population. Congenital lesions such as epidermoid and dermoid cysts and lipomas can also occur. Pineal gland tumors range from the well differentiated "pineocytoma"
(World Health Organization (WHO) grade I), with a good prognosis, to the aggressive poorly differentiated "pineoblastoma" (WHO grade IV) with "pineal parenchymal tumor of intermediate differentiation" (PPTID; WHO grades II and III) of intermediate differentiation and prognosis. Papillary tumor of the pineal region (PTPR; WHO grades II and III) The diagnostic criteria to differentiate these entities, especially between WHO grades II and III of both PPTID and PTPR still has grey zones.

Diagnosis of pineal region neoplasms is based on clinical presentation, imaging and HPE results. Serum and CSF biomarkers complement these standard diagnostic techniques by providing additional information before invasive procedures are carried out.

\section{Materials and Methods}

Cases of pineal region tumours received at a tertiary care hospital over a one year period were evaluated. Intraoperative cytology; frozen section and histopathology of the resected tumor were carried out. Wet-fixed (in 95\% ethanol) smears also were prepared and subsequently stained with Leishman Giemsa and Papanicolaou stain. For three of the patients an infratentorial supracerebellar approach was used for surgery whereas for one occipital transtentorial approach was used Paraffin embedded 3-4 micron sections were used for Haematoxylin and eosin stained sections, and relevant Immunohistochemical studies. IHC studies were carried out using LSAB (Labeled Streptavidin Biotin) technique. For assessing the proliferation index MIB1 labelling index was used using mouse 
antihuman MIB -1 MAb (Dako) (diluted $1: 300$ ) by manually counting 1000 cells from five representative fields at 400X. The cytology and histopathology findings were then correlated.

\section{Results and Discussion}

Though the incidence of pineal tumors in literature is $1 \%$ analysis of our database over the last one year revealed an incidence of $5.8 \%$ of all CNS tumours, The age varied between 5 to \%) years with a mean of 21.8 years. The male to female ratio was $1: 2$. Out of the five cases we encountered there were two pineocytomas, and one case each of PPTID, teratoma, and germinoma.

\section{Individual cases}

\section{Case 1}

45 yr Female presented with generalized headache and hydrocephalus. Figure $1(\mathrm{a}, \mathrm{b})$ reveal the imaging findings. On cytology there were small cells with relatively high $\mathrm{N} / \mathrm{C}$ ratios arranged in small clusters. A sheet of tumor cells showed relatively scant cytoplasm with clear borders, round to oval nuclei of uniform size, containing fine chromatin and small nucleoli. Rosette-like structures were occasionally found. On HPE the tumor was seen to be composed of cytologically bland isomorphic cells with uniform nuclear size, regular nuclear membranes and bland chromatin with low mitotic activity. These cells were found to be forming the characteristic pineocytomatous/neurocytic rosettes which is an irregular flower like arrangement of cells with a large meshwork of neuropil at the centre differing from Homer Wright rosettes by having more neuropil at the centre and the edges of the neuropil being undulating.IHC studies showed the tumor cells to be positive for Synaptophysin, chromogranin and NSE and the MIB 1 labeling index was $0.6 \%$. Based on this the diagnosis of Pineocytoma WHO grade I was confirmed.

\section{Case 2}

15 yr Male Clinical features with generalized headache and Parinaud's syndrome Serum alpha fetoprotein (AFP) Carcinoembryonic antigen (CEA) and human chorionic gonadotropin (hCG) were within normal limits. The imaging findings are shown in figure $2 \mathrm{a}$ and $2 \mathrm{~b}$ and on cytology there were large tumor cells accompanied by mature lymphocytes in the background, suggesting the two-cell pattern. The tumor cells had a round or oval nucleus with fine granular chromatin, one or a few prominent nucleoli and indistinct cell borders. Histopathology of the tumor showed uniform population of large round cells with vesicular nuclei and clear eosinophilic cytoplasm resembling germ cells admixed with lymphocytes which were CK positive. Based on this a diagnosis of Germinoma was given.

\section{Case 3}

5 years boy presented with gait Ataxia and hydrocephalus. The representative imaging findings are shown in figure $3 \mathrm{a}$ and $3 \mathrm{~b}$.

Cytology showed monolayered sheets of bland-looking cuboidal cells (root sheath elements) swirling sheets and aggregates of spindle-shaped cells (mesenchymal elements) with hyperchromatic nuclei, with scattered anucleate squames. Grossly the tumor showed a Lobulated neoplasm with a complex mixture of adult-type tissues from all three embryonic germ layers mature. Stratified squamous epithelium and skin appendages were seen as the ectodermal component. The mesoderm contributed to the presence of cartilage. Glandular epithelium was seen as a part of the endodermal component. No incompletely differentiated tissue elements that resembling fetal tissue was seen and hence this was classified as a mature teratoma.

\section{Case 4}

Twelve year old girl with generalized headache for 6months, Parinaud's syndrome and Hydrocephalus. The representative imaging findings are shown in figure $4 \mathrm{a}$ and $4 \mathrm{~b}$.

Cytology revealed cells with increased nucleocytoplasmic ratio, hyperchromatic nuclei, and small rosette-like cell cluster but cellular pleomorphism was mild to moderate and no necrosis was seen. Histopathology of this tumor showed a cellular tumor showing with diffuse sheets of uniform cells and the formation of small rosettes, with features intermediate between those of pineocytoma and those of pineoblastoma. Mitotic activity is was low being $0-1 / 10 \mathrm{Hpf}$. No of vascular proliferation or necrosis was seen. IHC showed that tumor was positive for both Synaptophysin, NSE and was GFAP Negative. A diagnosis of Pineal parenchymal tumor Grade-II was given. These tumors may fall into category II or III by the WHO classification. Some authors suggest that tumor with more than six mitotic figures per $10 \mathrm{HPF}$ and having NF positive should be graded as grade III. Molecular studies have revealed gains on 4qand $12 q$ and losses on 22 in these tumours however these were not carried out.

\section{Discussion}

A diverse variety of tumour histological types occur in the pineal gland these should be classified according to the latest edition of the WHO classification. Tumours of the pineal parenchyma are rare with incidence ranging between $0.2 \%$ and $2 \%[1,2]$. Signs and symptoms of pineal region masses are most often related to mass effect on the adjacent structures, patients usually present with headache, nausea, and vomiting as a result of increased intracranial pressure. Precocious puberty is more commonly associated with germ cell tumors (GCTs) and may be related to increased beta human chorionic gonadotropin (hCG) secreted by the tumor. Parinauds syndrome which comprises of vertical gaze paralysis, impaired papillary light reflex and convergence nystagmus as a result of compression or invasion of the tectal plate may at times be a presenting feature. Hemorrhage into a pineal tumor or cyst is referred to as pineal apoplexy the most common presenting symptom of which is a sudden decrease in consciousness associated with headache [4-6].

The commonest symptoms we encountered were diplopia; hydrocephalus and ataxia. Two patients also had features of Parin- 
aud's syndrome.

Modern neuro-imaging has greatly contributed to a more precise pre-operative diagnosis of intracranial tumors including pineal area tumors. MRI is the optimum modality although CT scanning is more specific about the presence of calcification. The correct interpretation of imaging should take into account the MRI appearance of normal anatomy of the pineal area and of that of other benign lesions typical in these sites. Germinoma, pineoblastoma, and Meningioma have high attenuation at CT due to their high cellularity, but if pineal calcification is seen the germinoma will tend to surround it, whereas in pineoblastomas it will be exploded to the peripheral areas. Meningioma on the other hand have a broad attachment to the dura. The diffusion is reduced in epidermoid cysts and may be present in germinoma and pineoblastomas. However, epidermoid cysts do not have internal enhancement unless there is rare development of squamous cell carcinoma. The presence of lipid or fat attenuation at CT leads to the differential diagnosis of a teratoma, dermoid cyst, or lipoma. Cerebrospinal fluid (CSF) seeding may be present at the time of presentation, especially in pineoblastomas and the higher grade PPTIDs [4,5]. Pineal gland tumors range from the well differentiated "pineocytoma" (World Health Organization (WHO) grade I), with a good prognosis, to the aggressive poorly differentiated "pineoblastoma" (WHO grade IV) with "pineal parenchymal tumor of intermediate differentiation" (PPTID; WHO grades II and III) of intermediate differentiation and prognosis and Papillary tumor of the pineal region (PTPR; WHO grades II and III).

Pineocytoma is a WHO grade I tumor, which affects young adults without gender difference. It is usually a solid mass, can sometimes show hemorrhagic or cystic appearance; it is highly differentiated and well circumscribed, with a low growth rate and rarely metastasizing.

The pineal parenchymal tumors include the pineocytoma WHO grade I, the pineoblastoma WHO grade IV and the pineal parenchymal tumour of intermediate differentiation. PPTID has intermediate histological features and is potentially aggressive. It corresponds to WHO grade II to III, but definite grading criteria have not been established, so that there is a need to refine prognostication for this tumour type. The current 2016 WHO classification has also not set any clearly defined criteria (like the number of mitoses) to distinguish between grades II and III of PPTIDs and also of PPTRs. Some Studies have used MIB1 LI, NFP expression and mitotic count, for grading of PPTID $[6,7]$.

Immunohistochemistry may also help with distinction from normal pineal, which has a MIB1 labelling index of 0 and a lobular pattern highlighted by GFAP. Low-grade pineal tumors can oc- casionally show cytological pleomorphism which can be a worrying feature. However, this feature alone (without elevated mitotic activity) does not appear to be associated with a worse prognosis and should not lead to upgrading. In 2000, Jouvet., et al. proposed a grading classification system for PPTs based on the architecture, mitotic figures, and immunostaining with neurofilament [8].

An array of germ cell tumors can develop in the pineal gland, mostly in younger subjects. Among germ cell tumors, teratomas, dermoid tumors, epidermoid tumors, and are considered benign; choriocarcinomas, endodermal sinus tumors, and embryonal carcinomas are considered malignant; and germinomas and immature teratomas fall in between. Patients with pineal area tumors should have serum sampled for AFP and B-HCG pre-operatively as in some cases a diagnosis of secreting germ-cell tumour can be made on tumour markers alone. Although mild elevation of $\beta$-HCG can be found in pure germinoma, marked elevation is associated with choriocarcinoma and embryonic carcinoma $[9,10]$. There is some debate over the $\beta$-HCG level that should be considered a marked elevation, with most authors suggesting that serum or CSF level greater than $50 \mathrm{~m} \mathrm{IU} / \mathrm{ml}$ places the patient in a high-risk or mixed germ cell category. B-hCG levels in germinomas are inconsistent, and germinomas with elevated $\beta$-hCG may have a poor prognosis $[11,12]$. However, in our study $\beta$-hCG was not raised in the one case of germinoma we encountered.

Immunohistochemical investigations, including antibodies to alpha -fetoprotein, human chorionic gonadotropin, placental alkaline phosphatase, and cytokeratins, c-kit (CD117), OCT4 and CD30 are of value for their diagnosis and subclassification.

Some germinomas develop a florid lymphoid or granulomatous inflammatory reaction that can suggest a differential diagnosis of an inflammatory process. The possibility of an underlying germinoma should therefore be considered in pineal biopsies showing inflammatory changes, and Immunohistochemistry is valuable to identify the hidden germinoma tumour cells $[9,10]$.

In addition to a number of other entities that can arise in this region, tumors can occasionally metastasize to the pineal region.

CSF cytology helps in diagnosis of leptomeningeal disease at both presentation or recurrence of tumours and therefore is of use and in diagnosis and staging. Immunocytochemistry can be performed on cytological preparations as well. Table 1 shows the radiological, cytological, histopathological features of pineal region tumours. 

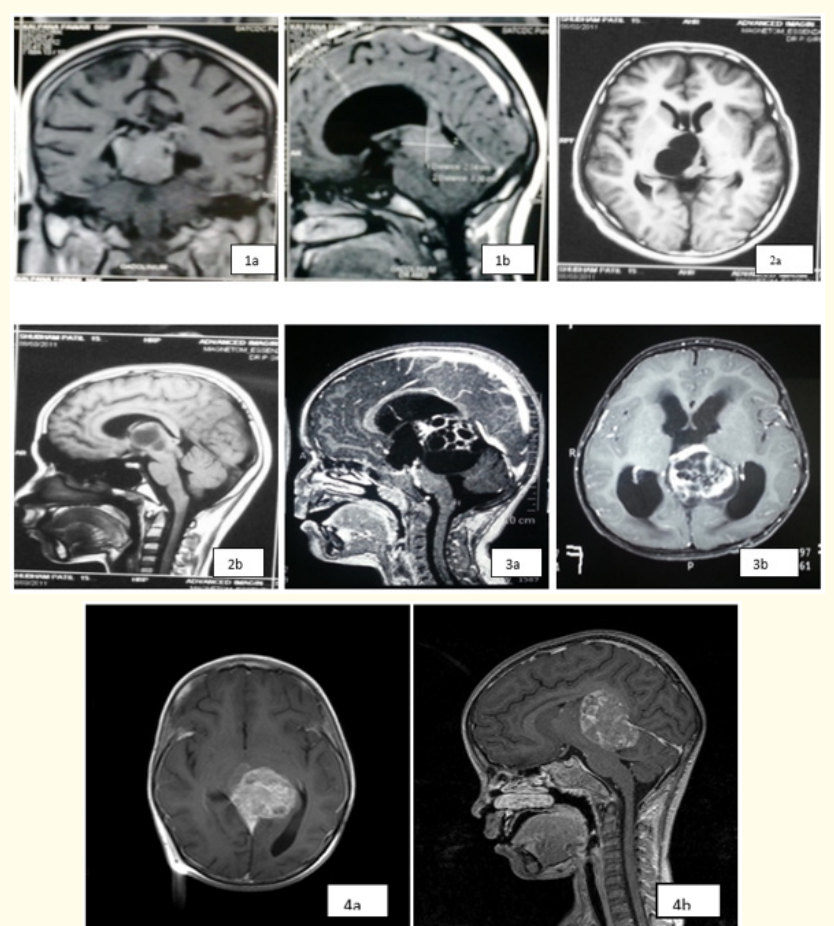

Figure A: Figure 1 (a, b): Pineocytoma WHO Grade 1, A Wellcircumscribed lesion in the pineal region displacing the internal cerebral vein superiorly and the midbrain and pons anteriorly. On post contrast images, it is showing homogeneous enhancement. Associated hydrocephalus is also noted. The pineal gland is not seen separately from the mass lesion. There is no significant heterogeneity within the mass. There are no features of Cystic or partially cystic components (no enhancing on post contrast images).

Figure 2 (a, b): Germinoma A midline solid cystic mass lesion (significant cystic component) seen in the pineal region. The epicenter of the mass is anterior and superior to the tectal plate. The tectal plate is seen separately from the mass lesion. There is no internal T1 hyperintensity suggestive of fat/calcification. The solid part of the mass is isointense to gray matter on T1-weighted images and demonstrate avid, homogeneous enhancement on post contrast images. Enhancing septations within the mass is also noted. Incidental note made of persistent cavum septum pellucidum. There is mass effect on the foramen of Monro. The mass is located predominantly in the midline and on the right side. There is no significant hydrocephalus.

Figure 3 (a, b) Mature teratoma: The pineal region solid cystic mass lesion has caused obstructive hydrocephalus. The pineal gland is not seen separately from the mass lesion. The soft-tissue component demonstrates enhancement on postcontrast images. The mass appears to be multilocular and shows areas of internal enhancement

Figure 4 (a, b) PPTID The pineal region mass is seen to displace the brainstem anteriorly. There is no significant hydrocephalus. The solid areas of the mass demonstrate postcontrast enhancement. Predominant cystic areas also noted. None of the imaging features are suggestive of any specific diagnosis. The visualized cervical cord in the visualized field of view appears normal (there is no evidence of drop metastasis).
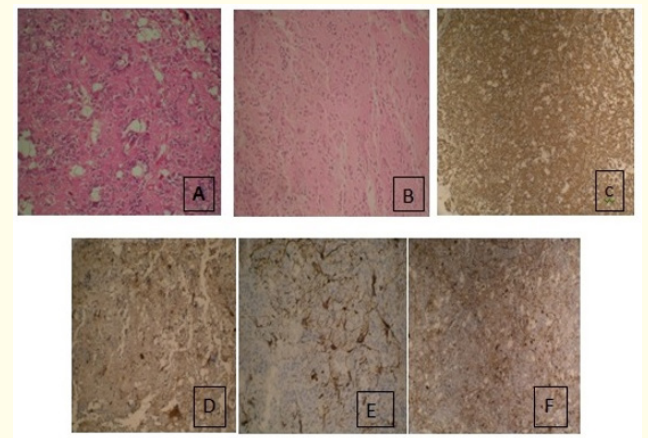

Figure 1: (A-E): A (HE 400X) Cells with high N/C ratios arranged in small clusters with scant cytoplasm with clear borders, round to oval nuclei of uniform size, containing fine chromatin and small nucleoli. Tumor cells showing positivity for Synaptophysin (C),

chromogranin (D), NSE (F) and negativity for GFAP(E).
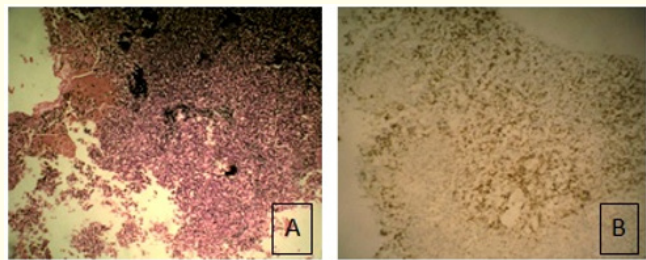

Figure 2: (A) Germinoma cells with watery clear cytoplasm accompanied by mature lymphocytes 2 (B) Tumor cells showing diffuse CK positivity.

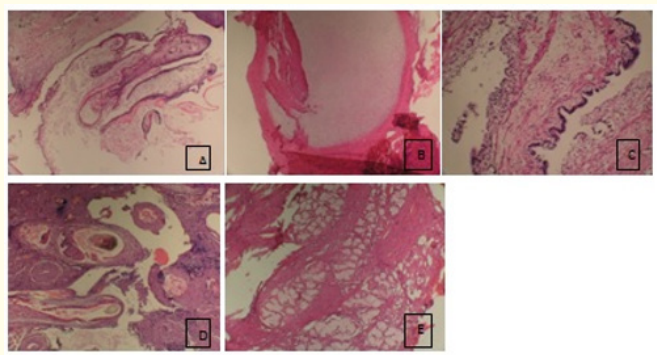

Figure 3: (A-E): Mature teratoma showing elements from all germ layers: Epidermal inclusion cyst (A) Cartilage (B) Respiratory Epithelium(C) Stratified squamous epithelium (D) Glands (E).
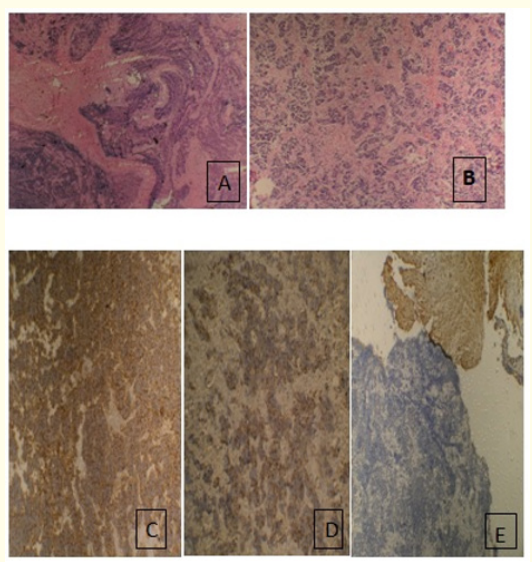

Figure 4: (A-E): (A) (Diffuse sheets of uniform cells with rosettes, with features interme $\neg$ diate between those of pineocytoma and those of pineoblastoma tumor cells, Tumor cells forming rosettes (B 400X) Tumor cells showing positivity for Synaptophysin (C), Chromogranin (D) and negativity for GFAP (E). 


\begin{tabular}{|c|c|c|c|c|}
\hline Tumour & Radiology & Cytology & Histopathology & IHC \\
\hline Pineoblastoma & $\begin{array}{l}\text { Large, lobulated, hyperat- } \\
\text { tenuating mass, most have } \\
\text { obstructive hydrocephalus } \\
\text { CSF dissemination common }\end{array}$ & $\begin{array}{l}\text { Small round blue primitive-ap- } \\
\text { pearing cells, nuclear molding, } \\
\text { mitoses/karyorrhexis+ necro- } \\
\text { sis+ }\end{array}$ & $\begin{array}{l}\text { Densely packed cells, high } \\
\text { grade hyperchromatic } \\
\text { nuclei, necrosis, mitotic } \\
\text { figures + Homer Wright, } \\
\text { Flexner-Wintersteiner } \\
\text { rosettes }\end{array}$ & $\begin{array}{l}\text { NSE+ } \\
\text { Syanptophysin+ } \\
\text { Retinal S antigen+ }\end{array}$ \\
\hline Meningioma & $\begin{array}{l}\text { Hyperattenuating, } \\
\text { strong and homogenous } \\
\text { enhancement on MRI with } \\
\text { contrast, dural tail }\end{array}$ & $\begin{array}{l}\text { Whorls and syncytia of bland } \\
\text { fibroblastic-type cells } \\
\text { with fusiform nuclei }\end{array}$ & $\begin{array}{l}\text { Meningothelial whorls, } \\
\text { syncytial cells indistinct } \\
\text { cell membranes psammo- } \\
\text { ma bodies, meningothelial } \\
\text { whorls }\end{array}$ & $\begin{array}{l}\text { Vimentin+, EMA +, } \\
\text { GFAP-ve }\end{array}$ \\
\hline Pineal cyst & $\begin{array}{l}\text { Midline, well circumscribed, } \\
\qquad 1-3 \mathrm{~cm}\end{array}$ & $\begin{array}{l}\text { Scant cellularity, reactive glial } \\
\text { cells and few benign } \\
\text { polygonal parenchymal cells. }\end{array}$ & $\begin{array}{l}\text { Glial tissue, pineal paren- } \\
\text { chyma, connective tissue }\end{array}$ & \\
\hline Epidermoid cyst & $\begin{array}{l}\text { Low attenuation, rim en- } \\
\text { hancement may rarely be } \\
\text { seen and reflects inflamma- } \\
\text { tion or infection, but there } \\
\text { is no internal enhancement, } \\
\text { unlike in teratomas }\end{array}$ & $\begin{array}{l}\text { Mature squamous cells, granu- } \\
\text { lar debris }\end{array}$ & $\begin{array}{c}\text { Simple stratified squamous } \\
\text { epithelium, }\end{array}$ & $\mathrm{CK}+$ \\
\hline
\end{tabular}

Table 1: Highlights of radiological, cytological, histopathological features of pineal region tumours.

\section{Conclusion}

To conclude Workup of a pineal mass presently entails imaging followed by serum and CSF laboratory workup for germ cell tumor markers. Imaging and laboratory workup are followed by biopsy.
The standard treatment for pineal parenchymal tumors is radiation. Surgery is an option, particularly for children who often have adverse consequences from chemotherapy or radiation. However, surgery has a mortality rate of 5\%-10\%. 


\section{Conflict of Interest}

No financial interest or any conflict of interest exists.

\section{Bibliography}

1. Jouvet A., et al. "Pineal parenchymal tumours and pineal cysts". Neurochirurgie 61.2-3 (2015): 123-129.

2. Ostrom Quinn T., et al. "CBTRUS statistical report: primary brain and other central nervous system tumors diagnosed in the United States in 2009-2013". Neuro-oncology 18.5 (2016): $1-75$.

3. Villano J Lee, et al. "Malignant pineal germ-cell tumors: an analysis of cases from three tumor registries". Neuro-oncology 10.2 (2008): 121-130.

4. Osborn AG., et al. "The new World Health Organization classification of central nervous system tumors: what can the neuroradiologist really say?". American Journal of Neuroradiology 33.5 (2012): 795-802.

5. Fèvre-Montange M., et al. "Utility of Ki67 immunostaining in the grading of pineal parenchymal tumours: a multicentre study". Neuropathology and Applied Neurobiology 38.1 (2012): 87-94.

6. Jouvet Anne., et al. "Pineal parenchymal tumors: a correlation of histological features with prognosis in 66 cases". Brain Pathology 10.1 (2000): 49-60.

7. Sugiyama K., et al. Intracranial germ-cell tumor with synchronous lesions in the pineal and suprasellar regions: report of six cases and review of the literature". Surgery in Neurology 38.2 (1992): 114-121.

8. Nagasawa Daniel T., et al. "Pineal germ cell tumors: two cases with review of histopathologies and biomarkers". Journal of Clinical Neuroscience 38 (2017): 23-31.

9. Schild Steven E., et al. "Pineal parenchymal tumors: clinical, pathologic, and therapeutic aspects". Cancer 72.3 (1993): 870880.

10. De Girolami U., et al. "Pathology of tumors of the pineal region”. Revue Neurologique 164.11 (2008): 882-895.

11. Villa Salvador., et al. "Primary pineal tumors: outcome and prognostic factors-a study from the Rare Cancer Network (RCN)". Clinical and Translational Oncology 14.11 (2012): 827834.

12. Schild Steven E., et al. "Histologically confirmed pineal tumors and other germ cell tumors of the brain". Cancer: Interdisciplinary International Journal of the American Cancer Society 78.12 (1996): 2564-2571.

\section{Assets from publication with us}

- Prompt Acknowledgement after receiving the article

- Thorough Double blinded peer review

- Rapid Publication

- Issue of Publication Certificate

- High visibility of your Published work

Website: www.actascientific.com/

Submit Article: www.actascientific.com/submission.php

Email us: editor@actascientific.com

Contact us: +919182824667 